\title{
Efficient Preconditioning of the Method of Auxiliary Sources (MAS) for Cylindrical Scatterers of Quasi- Circular Cross-Section
}

\author{
Hristos T. Anastassiu*, ${ }^{*}$, Georgios K. Avdikos ${ }^{2}$ and Angelos T. Vouldis ${ }^{3}$ \\ ${ }^{I}$ Hellenic Aerospace Industry S. A., Department of Communications and Networks Design and Development, P.O. Box \\ 23, GR-32009, Schimatari-Tanagra, Viotia, Greece \\ ${ }^{2}$ National Technical University of Athens, School of Applied Mathematics and Physics, and also with Raymetrics S. A., \\ Athens, Greece \\ ${ }^{3}$ National Technical University of Athens, School of Electrical and Computer Engineering, Athens, Greece
}

\begin{abstract}
Two different forms of a preconditioning process (i.e. standard preconditioning and quasi-diagonalization) are presented, in conjunction with the Method of Auxiliary Sources (MAS), when the latter is applied to a specific class of two-dimensional scattering problems. The method enhances the efficiency of MAS, when the linear system becomes illconditioned, due to distancing of the auxiliary surface from the outer boundary. If the cross-sectional boundary is geometrically close to a circle, it is proven that the MAS matrix becomes quasi-circulant, as intuition dictates. By exploiting the properties of the exactly circulant matrix, pertaining to the original circular configuration, the perturbed system is transformed to a quasi-diagonal one, whose inversion is a numerically stable operation.
\end{abstract}

\section{INTRODUCTION}

It is well known that the performance of the Method of Auxiliary Sources (MAS) depends on the location of the auxiliary sources (ASs). If the latter is not carefully chosen, numerical inaccuracy may constitute a severe problem. This complication has been resolved so far only for a few canonical geometries (i.e. [1-6]). In general terms, the singularities of the scattered field should be enclosed by the auxiliary surface as tightly as possible. Although the exact location of these singularities is unknown for arbitrary geometries, it was shown in [7] that the radius of the smallest possible circle, or sphere (in $2 \mathrm{D}$ and $3 \mathrm{D}$ respectively), surrounding them all, can be determined analytically. An auxiliary surface, conformal to the scatterer surface, circumscribing this circle/sphere is expected to yield optimal results, i.e. minimize the boundary condition error. In the same way, for a given, sufficiently low boundary condition error, the optimal auxiliary surface will contain the minimum number of unknowns, which is of high significance when large scatterers are considered. From the results of [8] it is implied that this observation holds true indeed, for various geometries, including an elliptic cylinder, where scattered field singularities coincide with the foci. However, the actual error behavior in such a situation is usually unclear, due to additive numerical noise. As a matter of fact, when the AS's lie close to singularities, the condition number of the MAS linear system increases dramatically, and hence numerical inversion of the system may become inaccurate, leading to deterioration of the overall computational error. To alleviate this effect, matrix preconditioning is employed in this paper for several 2D

*Address correspondence to these authors at the Hellenic Aerospace Industry S. A., Department of Communications and Networks Design and Development, P.O. Box 23, GR-32009, Schimatari - Tanagra, Viotia, Greece; E-mail: Anastasiou.Christos@ haicorp.com configurations, which can be considered as perturbations of a circle.

If the geometry closely resembles an exact circle, the pertinent MAS matrix is found to be a perturbation of the circulant matrix, corresponding to that circle. Since inversion of the latter can be performed analytically [1], multiplication of its inverse with the original, perturbed matrix can be carried out with maximal accuracy. The product of the two will have the form of a perturbed identity matrix, which is naturally well- conditioned, and hence easily invertible from a numerical point of view. Alternatively, the concept of quasidiagonalization may be invoked. The original matrix is multiplied from the right with the eigenvector matrix of the circular case and from the left with its transpose (equal to its inverse). This bilateral multiplication yields an almost diagonal matrix. Its most significant elements lie on the diagonal, and are essentially perturbations of the eigenvalues of the circular case. Again, this resulting quasi-diagonal matrix is readily invertible via a numerical scheme, since the inversion procedure has been stabilized. Results are presented for several, circle-like geometries, validating the preconditioning concept, and leading to high precision MAS solutions of complex scattering problems. Preliminaries of this work have been presented in [9].

\section{STANDARD PRECONDITIONING}

In [1] it was proven that for the benchmark problem of scattering from a perfectly conducting (PEC) circular cylinder (see Fig. 1), the MAS matrix is circulant, and therefore diagonalizable and analytically invertible. Using the notation in [1] the elements of the MAS matrix are given by

$$
Z_{m n}^{(0)} \equiv-\frac{j}{4} \sum_{l=-\infty}^{\infty} J_{l}(k a) H_{l}^{(2)}(k b) \exp \left\{-j l\left(\phi_{m}-\phi_{n}^{\prime}\right)\right\}
$$


where $b$ is the cylinder radius, $a$ is the radius of the auxiliary surface, $k$ is the wavenumber, $J_{l}(\bullet)$ is the Bessel function of order $l, H_{l}^{(2)}(\bullet)$ is the Hankel function of the second kind and order $l, \phi_{m} \equiv m \cdot 2 \pi / N$ is the azimuth angle of the $m^{\text {th }}$ collocation point (CP), $\phi_{n}^{\prime} \equiv n \cdot 2 \pi / N$ is the azimuth angle of the $n^{\text {th }} \mathrm{AS}$ and $N$ is the number of ASs.

This matrix can be diagonalized as follows:

$\left[\boldsymbol{Z}^{(0)}\right]=[\boldsymbol{G}]\left[\boldsymbol{L}^{(0)}\right][\boldsymbol{G}]^{-1}$

where $\left[\boldsymbol{L}^{(0)}\right]$ is a diagonal matrix with elements equal to the eigenvalues of $\left[\boldsymbol{Z}^{(0)}\right]$, namely

$\lambda_{q}=-\frac{j N}{4} \sum_{s=-\infty}^{\infty} J_{q+s N}(k a) H_{q+s N}^{(2)}(k b) \quad q=1, \ldots, N$

and $[\boldsymbol{G}]$ is the unitary matrix (i.e. $[\boldsymbol{G}]^{-1}=[\boldsymbol{G}]^{k}$ ) containing all eigenvectors

$\{\boldsymbol{g}\}_{q}=\frac{1}{\sqrt{N}}\left[\exp \left\{-j q \phi_{1}\right\}, \exp \left\{-j q \phi_{2}\right\} \ldots, \exp \left\{-j q \phi_{N}\right\}\right]^{T}$

as consecutive columns. Essentially, $[\boldsymbol{G}]$ is the well-known Fourier matrix. As explained in [1], the MAS system is analytically invertible, a property attributed to the exact diagonalizability expressed by (2). Although (1)-(4) are exclusively applicable to circular cross-sections, in this paper it will be shown that they can be exploited indirectly even in the case of non-circular configurations.

To prove this claim, assume an infinite, cylindrical, PEC scatterer with a cross-section, which can be considered as a deformation (perturbation) of a circle (see Fig. 2). Let the analytical expression of the cross- sectional boundary be (in polar coordinates)

$\rho(\phi)=b+\Delta \rho(\phi)$

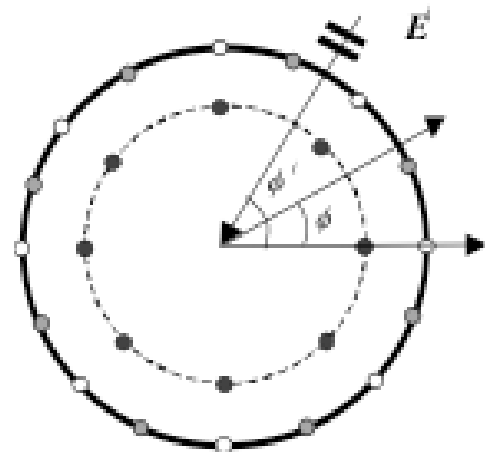

Fig. (1). Geometry of the problem. Black, white and gray bullets represent Auxiliary Sources (ASs), Collocation Points (CPs) and Midpoints (MPs) respectively.

where $b$ is the radius of the unperturbed circular geometry and $\Delta \rho(\phi)$ is the (relatively small) perturbation at an arbitrary angle $\phi$. At the MAS CPs $\phi_{m} \equiv m \cdot 2 \pi / N$ we set.

$\rho\left(\phi_{m}\right) \equiv \rho_{m}=b+\Delta \rho\left(\phi_{m}\right)=b+b \varepsilon_{m}=b\left(1+\varepsilon_{m}\right)$

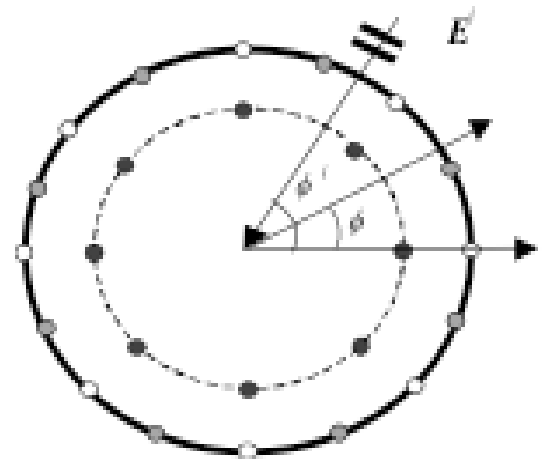

Fig. (2). Geometry of the perturbed problem. Black, white and gray bullets represent Auxiliary Sources (ASs), Collocation Points (CPs) and Midpoints (MPs) respectively.

where $\left|\varepsilon_{m}\right| \ll<1$. Similarly, for the auxiliary surface, which is again a deformed circle of original radius $a$, the radii of the auxiliary sources' locations are equal to

$$
\rho\left(\phi_{n}^{\prime}\right) \equiv \rho_{n}^{\prime}=a+\Delta \rho\left(\phi_{n}^{\prime}\right)=a+a \varepsilon_{n}=a\left(1+\varepsilon_{n}\right)
$$

where the $\varepsilon$ 's are the same in (6) and (7), since the auxiliary surface remains conformal (similar) to the outer boundary. The resulting MAS matrix of the perturbed problem, with elements given by

$$
Z_{m n} \equiv-\frac{j}{4} \sum_{l=-\infty}^{\infty} J_{l}\left(k \rho_{n}^{\prime}\right) H_{l}^{(2)}\left(k \rho_{m}\right) \exp \left\{-j l\left(\phi_{m}-\phi_{n}^{\prime}\right)\right\}
$$

is no longer circulant, and hence the diagonalization procedure described in [1]-[6] is not directly applicable to this case. However, via Taylor expansion

$J_{l}\left(k \rho_{n}^{\prime}\right)=J_{l}(k a)+k a \varepsilon_{n} \dot{J}_{l}(k a)+\frac{\left(k a \varepsilon_{n}\right)^{2}}{2} \ddot{J}_{l}(k a)+\ldots$

where the dot denotes differentiation with respect to the entire argument. Similarly,

$$
\begin{aligned}
& H_{l}^{(2)}\left(k \rho_{m}\right)=H_{l}^{(2)}(k b)+k b \varepsilon_{m} \dot{H}_{l}^{(2)}(k b)+ \\
& +\frac{\left(k b \varepsilon_{m}\right)^{2}}{2} \ddot{H}_{l}^{(2)}(k b)+\ldots
\end{aligned}
$$

Up to the first order in $\varepsilon$ 's, the MAS matrix can be approximated, due to (9) and (10) as

$$
[\boldsymbol{Z}] \cong\left[\boldsymbol{Z}^{(0)}\right]+k a[\boldsymbol{E}]\left[\boldsymbol{Z}^{(1)}\right]+k b\left[\boldsymbol{Z}^{(2)}\right][\boldsymbol{E}]
$$

where

$$
\begin{aligned}
& Z_{m n}^{(1)} \equiv-\frac{j}{4} \sum_{l=-\infty}^{\infty} \dot{J}_{l}(k a) H_{l}^{(2)}(k b) \exp \left\{-j l\left(\phi_{m}-\phi_{n}^{\prime}\right)\right\} \\
& Z_{m n}^{(2)} \equiv-\frac{j}{4} \sum_{l=-\infty}^{\infty} J_{l}(k a) \dot{H}_{l}^{(2)}(k b) \exp \left\{-j l\left(\phi_{m}-\phi_{n}^{\prime}\right)\right\}
\end{aligned}
$$

and

$$
[E] \equiv \operatorname{diag}\left[\varepsilon_{1}, \varepsilon_{2}, \ldots, \varepsilon_{N}\right]
$$


Obviously, $\left[\boldsymbol{Z}^{(1)}\right]$ and $\left[\boldsymbol{Z}^{(2)}\right]$ are also circulant, and can therefore be diagonalized in a manner analogous to (2). The eigenvectors remain the same as in (4), and the eigenvalues are similar to (3), the dot being the only difference.

Let the original MAS system be written as

$[\boldsymbol{Z}]\{\boldsymbol{J}\}=\{\boldsymbol{V}\}$

where $\{\boldsymbol{J}\}$ is the column vector of the unknowns and $\{\boldsymbol{V}\}$ contains the samples of the incident field [1]. Using (11) and multiplying from the left by $\left[\boldsymbol{Z}^{(0)}\right]^{-1},(15)$ can be written as

$$
\begin{aligned}
& \left([\boldsymbol{I}]+k a\left[\boldsymbol{Z}^{(0)}\right]^{-1}[\boldsymbol{E}]\left[\boldsymbol{Z}^{(1)}\right]+k b\left[\boldsymbol{Z}^{(0)}\right]^{-1}\left[\boldsymbol{Z}^{(2)}\right][\boldsymbol{E}]\right)\{\boldsymbol{J}\} \cong \\
& \cong\left[\boldsymbol{Z}^{(0)}\right]^{-1}\{\boldsymbol{V}\}
\end{aligned}
$$

where $[\boldsymbol{I}]$ is the $N \times N$ identity matrix. Given the properties of the circulant matrices involved, (16) can be written explicitly as

$$
\begin{aligned}
& \left([\boldsymbol{I}]+k a[\boldsymbol{G}]\left[\boldsymbol{L}^{(0)}\right]^{-1}[\tilde{\boldsymbol{E}}]\left[\boldsymbol{L}^{(1)}\right][\boldsymbol{G}]^{-1}+\right. \\
& \left.+k b[\boldsymbol{G}]\left[\boldsymbol{L}^{(0)}\right]^{-1}\left[\boldsymbol{L}^{(1)}\right][\tilde{\boldsymbol{E}}][\boldsymbol{G}]^{-1}\right)\{\boldsymbol{J}\} \cong \\
& {[\boldsymbol{G}]\left[\boldsymbol{L}^{(0)}\right]^{-1}[\boldsymbol{G}]^{-1}\{\boldsymbol{V}\}}
\end{aligned}
$$

\section{where}

$$
[\tilde{\boldsymbol{E}}] \equiv[\boldsymbol{G}]^{-1}[\boldsymbol{E}][\boldsymbol{G}]
$$

and $\left[\boldsymbol{L}^{(0,1,2)}\right]$ are diagonal matrices with elements equal to the eigenvalues of $\left[\boldsymbol{Z}^{(0,1,2)}\right]$. In (17) the matrix on the left hand side, defined here for brevity as $[\boldsymbol{K}] \equiv\left[\boldsymbol{Z}^{(0)}\right]^{-1}[\boldsymbol{Z}]$, is readily identifiable as a perturbation of the identity matrix. Since $\left|\varepsilon_{m}\right|<<1$, the magnitudes of the second and third terms in the system matrix are generally much smaller than the diagonal elements, due to the strongly diagonal character of $[\boldsymbol{I}]$. Therefore, (17) is a much better conditioned system than (15), and hence it is numerically tractable. Thus, the preconditioning proposed herein, can be performed via left multiplication of the original system by $\left[\boldsymbol{Z}^{(0)}\right]^{-1}$, which is known analytically, due to (2). Furthermore, (2) facilitates an easy implementation of this multiplication, since only diagonal and Fourier matrices are involved in the calculations.

Although the inversion of the MAS matrix has been stabilized, accurate calculation of the auxiliary currents $\{\boldsymbol{J}\}$ may still cause problems for very small auxiliary surfaces. This is due to the occurrence of very small eigenvalues in the unperturbed, circulant matrix, when the auxiliary surface shrinks too much, i.e. when approaching the origin. In that case, even inversion of the diagonal $\left[\boldsymbol{L}^{(0)}\right]$ in (17) is numerically challenging, since a few of its entries are vanishingly small. Inaccuracies are observed again, and apparently not much has been gained after all. However, the objective in a scattering problem is the calculation of the scattered field, and not the auxiliary currents, which is only an intermediate step (see [10] for analogous reasoning in a slightly different topic). The field can still be accurately calculated, by artificially cancelling out any exceedingly large matrix entries. To demonstrate how this can be achieved, let us calculate the scattered field at the midpoints (MPs) of the scattering surface, in a way similar to [1]. Calculation of these values will also reveal the behavior of the boundary condition error [1].

Let the samples of the scattered (i.e. radiated from the ASs) field at the MPs be contained in $\{\hat{\boldsymbol{V}}\}$. Then, according to [1],

$$
\{\hat{\boldsymbol{V}}\}=[\hat{\boldsymbol{Z}}]\{\boldsymbol{J}\}
$$

where

$$
\widehat{Z}_{m n} \equiv-\frac{j}{4} \sum_{l=-\infty}^{\infty} J_{l}\left(k \rho_{n}^{\prime}\right) H_{l}^{(2)}\left(k \widehat{\rho}_{m}\right) \exp \left\{-j l\left(\widehat{\phi}_{m}-\phi_{n}^{\prime}\right)\right\}
$$

and $\left(\widehat{\rho}_{m}, \widehat{\phi}_{m}\right)$ is the location of the $m^{\text {th }}$ MP. Evidently, $[\widehat{Z}]$ is amenable to a perturbation analysis similar to (9)(14). Let $\left[\hat{\boldsymbol{Z}}^{(0)}\right]$ be the corresponding circulant matrix, $\left[\widehat{\boldsymbol{L}}^{(0)}\right]$ the diagonal matrix containing the eigenvalues of $\left[\widehat{\boldsymbol{Z}}^{(0)}\right]$, and let $[\hat{\boldsymbol{K}}] \equiv\left[\widehat{\boldsymbol{Z}}^{(0)}\right]^{-1}[\hat{\boldsymbol{Z}}]$. Then, from (16), (17) and (19) it follows that

$$
\{\widehat{\boldsymbol{V}}\}=[\boldsymbol{G}]\left[\widehat{\boldsymbol{L}}^{(0)}\right][\boldsymbol{G}]^{-1}[\hat{\boldsymbol{K}}][\boldsymbol{K}]^{-1}[\boldsymbol{G}]\left[\boldsymbol{L}^{(0)}\right]^{-1}[\boldsymbol{G}]^{-1}\{\boldsymbol{V}\}
$$

From (21), it can be seen that $\{\hat{\boldsymbol{V}}\}$ can be accurately calculated, irrespective of vanishingly small eigenvalues. Indeed, $\quad[\hat{\boldsymbol{K}}][\boldsymbol{K}]^{-1}$ is nearly an identity matrix, $[\boldsymbol{G}]^{-1}[\widehat{\boldsymbol{K}}][\boldsymbol{K}]^{-1}[\boldsymbol{G}]$ likewise, and hence, when $\left[\widehat{\boldsymbol{L}}^{(0)}\right][\boldsymbol{G}]^{-1}[\widehat{\boldsymbol{K}}][\boldsymbol{K}]^{-1}[\boldsymbol{G}]\left[\boldsymbol{L}^{(0)}\right]^{-1}$ is calculated, very small entries on the diagonal of $\left[\widehat{\boldsymbol{L}}^{(0)}\right]$ almost cancel out the very large entries on the diagonal of $\left[\boldsymbol{L}^{(0)}\right]^{-1}$, thus circumventing the problem reported before. The numerical results will demonstrate the validity of this procedure.

\section{QUASI-DIAGONALIZATION}

Alternatively, another convenient transformation of the original linear system can be derived as follows: Multiplying (15) from the left by $[G]^{-1}$ yields

$$
[G]^{-1}[\boldsymbol{Z}][G][G]^{-1}\{\boldsymbol{J}\}=[G]^{-1}\{\boldsymbol{V}\}
$$

Due to (11), (22) can be written as

$$
\left(\left[L^{(0)}\right]+k a[G]^{-1}[\boldsymbol{E}]\left[\boldsymbol{Z}^{(1)}\right][G]+k b[G]^{-1}\left[\boldsymbol{Z}^{(2)}\right][\boldsymbol{E}][G]\right) \bullet
$$




$$
\bullet\left([G]^{-1}\{\boldsymbol{J}\}\right) \cong\left([G]^{-1}\{\boldsymbol{V}\}\right)
$$

The square matrix on the left hand side is evidently a perturbation of the diagonal matrix $\left[L^{(0)}\right]$, thus the procedure can be named as "quasi-diagonalization". Solving (23) for $[G]^{-1}\{\boldsymbol{J}\}$ is a numerically stable operation, although the solution of the original system may not necessarily be. Finally, retrieval of $\{\boldsymbol{J}\}$ from $[G]^{-1}\{\boldsymbol{J}\}$ is trivially performed through multiplication by $[G]$.

\section{NUMERICAL RESULTS}

Several configurations were investigated numerically, to validate the mathematical concepts of the previous sections. First, an elliptic, infinite, PEC cylinder with ellipticity $\gamma=0.95$ and maximum radius $b=\lambda$ was considered, and plots of the system condition number (in the 1-norm) before and after preconditioning and quasi-diagonalization are depicted

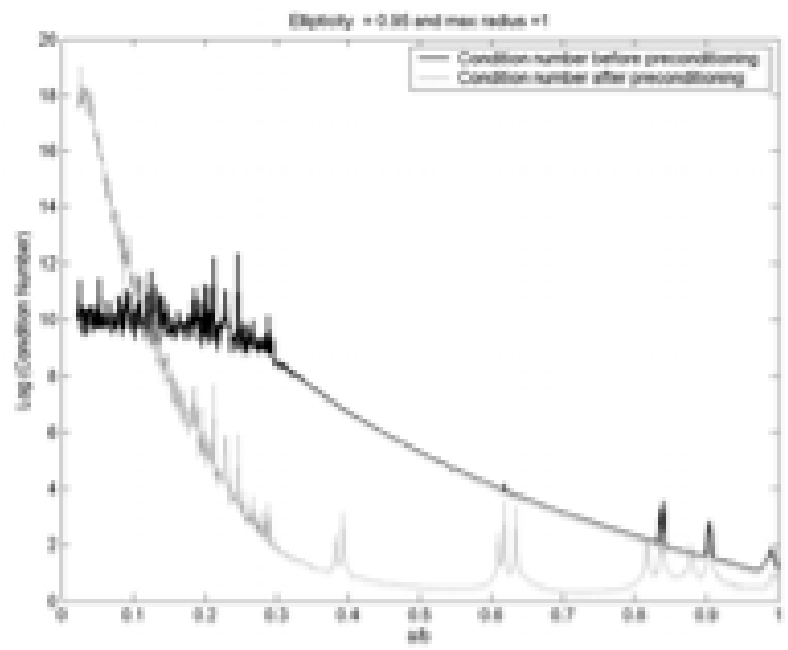

Fig. (3). Condition number for the MAS system of the elliptic cylinder with $\gamma=0.95$, maximum radius $b=\lambda$, and $N=30$, before and after standard preconditioning.

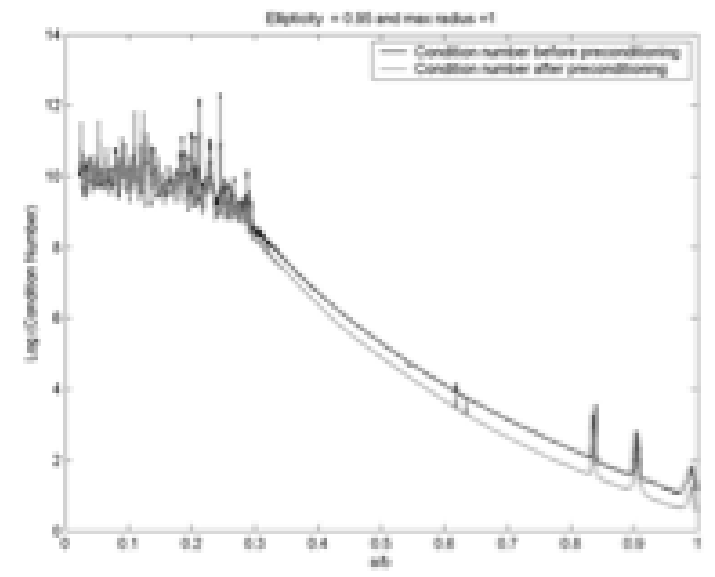

Fig. (4). Condition number for the MAS system of the elliptic cylinder with $\gamma=0.95$, maximum radius $b=\lambda$, and $N=30$, before and after quasi- diagonalization. in Figs. (3 and 4). It is evident that the system conditioning improves significantly, especially for standard preconditioning. Condition improvement may not be as spectacular for quasi-diagonalization, however even the small change shown in Fig. (4) affects the solution accuracy considerably. Indeed, the effect is reflected on Fig. (5), where the solution error (2norm of the difference between the left and the right hand side of the linear system) is plotted before and after quasidiagonalization. It should be emphasized that the foci of this ellipse lie at a distance equal to $0.31 \lambda$ from the origin, and therefore the MAS system is not capable of solving the scattering problem for very small auxiliary surfaces not enclosing these two points [7]. Hence, the deterioration of the condition number for small $a / b$, as seen in Figs. (3 and $\mathbf{4}$ ), does not have any consequence on the MAS solution of the problem, since this solution is meaningless, anyway. Figs. (6 and 7) depict the boundary condition error (in the sense of [1]) for an elliptic cylinder with $\gamma=0.995, N=30$ and $N=40$ respectively. The reduction of the boundary condition error is significant for a wide range of similarity ratios. Also, Fig. (8)

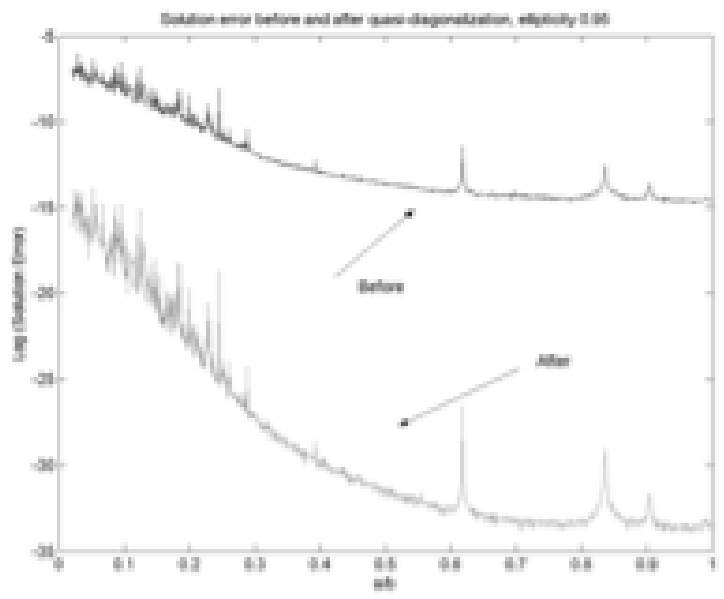

Fig. (5). Solution error for the MAS system of the elliptic cylinder with $\gamma=0.95$, maximum radius $b=\lambda$, and $N=30$, before and after quasi-diagonalization.

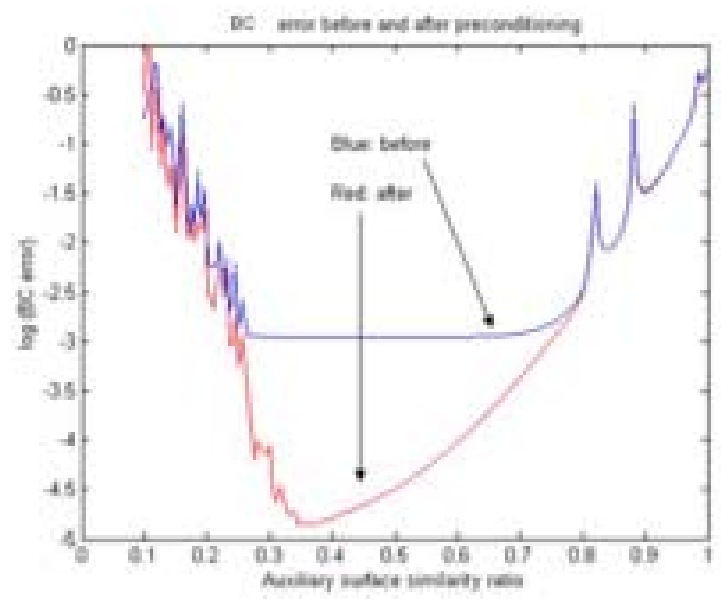

Fig. (6). Boundary condition error for the MAS system of the elliptic cylinder with $\gamma=0.995$, maximum radius $b=\lambda$, and $N=30$, before and after standard preconditioning. 


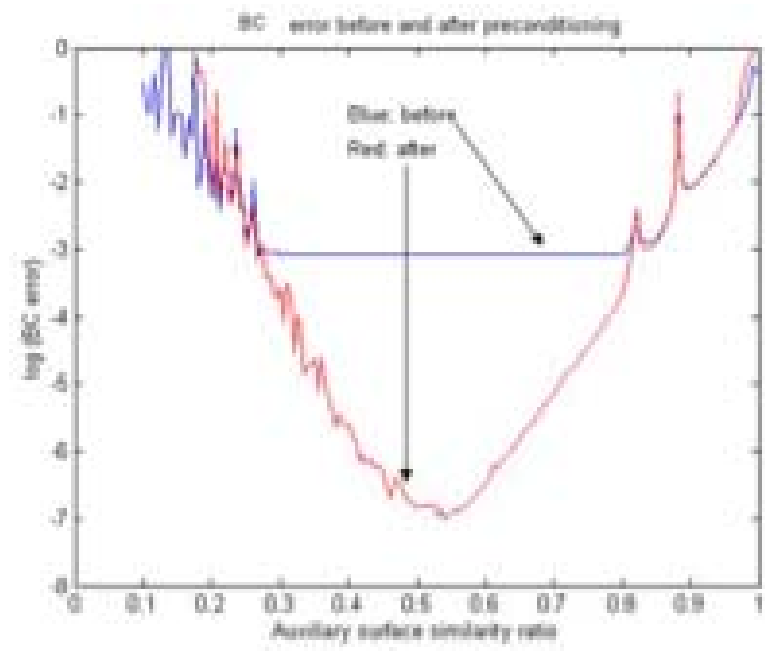

Fig. (7). Boundary condition error for the MAS system of the elliptic cylinder with $\gamma=0.995$, maximum radius $b=\lambda$, and $N=40$, before and after standard preconditioning.

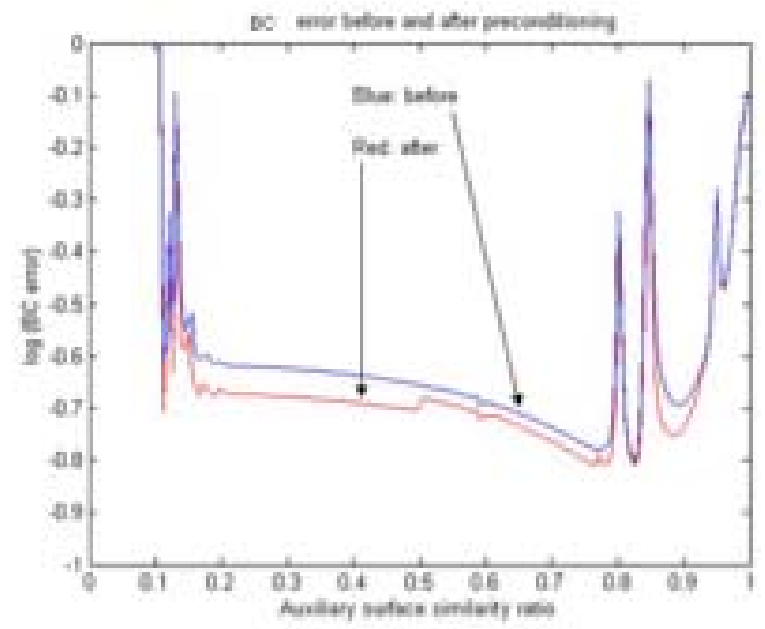

Fig. (8). Boundary condition error for the MAS system of the super-elliptic cylinder with $a=b=\lambda, v=2.5$ and $N=20$ before and after standard preconditioning.

presents the boundary condition error for a supper-elliptic cylinder (defined by $(x / a)^{v}+(y / b)^{v}=1$ ) with $v=2.5$ and $N=20$, before and after preconditioning. The reduction of the overall boundary condition error is visible in this case, too. Finally, Figs. (9, 10 and 11) depict the diagonally dominant behavior of the MAS matrix for the ellipse of Figs. (3-5), after preconditioning and quasi-diagonalization. As it can be easily seen, after enforcement of either type of preconditioning (standard or quasi-diagonalization), the matrix elements concentrate towards the diagonal, simultaneously increasing their magnitude. Therefore, in a loose sense, the matrix becomes "more diagonal", and hence the linear system becomes much easier to solve.

\section{CONCLUSIONS}

This paper proposed two different versions of a preconditioning technique, applicable to two-dimensional scattering

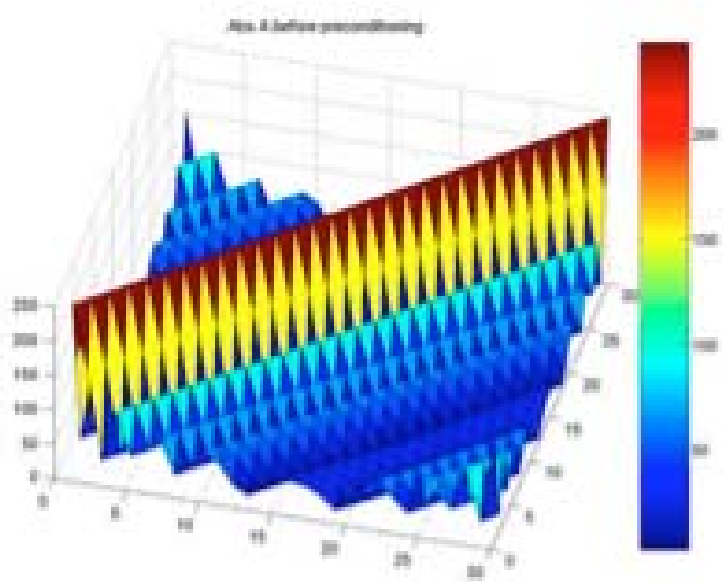

Fig. (9). Magnitude of the original matrix elements, corresponding to the geometry of Figs. (3-5).

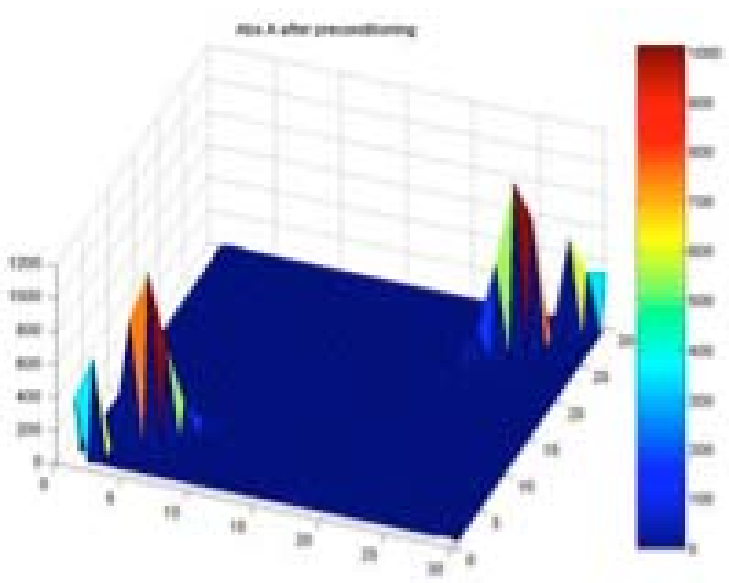

Fig. (10). Magnitude of the matrix elements corresponding to the geometry of Figs. (3-5), after standard preconditioning.

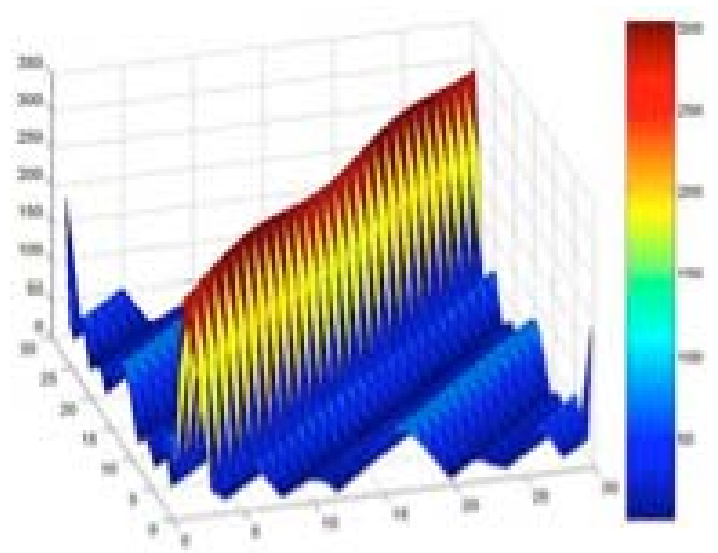

Fig. (11). Magnitude of the matrix elements, corresponding to the geometry of Figs. (3-5), after quasi-diagonalization

problems, being solved via MAS. It was shown that the fundamental concept of the procedure is the exploitation of the properties of the MAS circulant matrix, associated with the geometrically closest circular configuration. The original 
system, which is quasi-circulant (but not exactly circulant), can be transformed to a quasi-diagonal one, which demonstrates remarkable numerical stability during the inversion process. Several quasi-circular cylinders can hence be analyzed via MAS, disregarding possible ill-conditioning of the original system. Therefore, shrinking of the auxiliary surface away from the boundary of the geometry is feasible, since it is not affected by numerical noise, allowing accurate solutions based only on a very small number of unknowns.

\section{REFERENCES}

[1] H. T. Anastassiu, D. G. Lymperopoulos and D. I. Kaklamani, "Accuracy analysis and optimization of the Method of Auxiliary Sources (MAS), for scattering by a circular cylinder", IEEE Trans. Antennas Propagat., vol. 52, no. 6, June 2004, pp. 1541-1547.

[2] H. T. Anastassiu and D. I. Kaklamani, "Accuracy analysis and optimization of the Method of Auxiliary Sources (MAS), applied to TE scattering by a perfectly conducting circular cylinder", $J$. Electromag. Waves Appl., vol. 18, no. 10, 2004, pp. 1283-1294.

[3] H. T. Anastassiu and D. I. Kaklamani, "Error estimation of the Method of Auxiliary Sources (MAS), for scattering from a dielectric circular cylinder", Radio Sci., vol. 39, no. 5, RS5015, doi: 10.1029/2004RS003028, Oct. 2004
[4] H. T. Anastassiu, "Error estimation of the Method of Auxiliary Sources (MAS), for scattering from an impedance circular cylinder", Prog. Electromag. Res. (PIER), 52, 2005, pp. 109-128.

[5] N. L. Tsitsas, E. G. Alivizatos, H. T. Anastassiu and D. I. Kaklamani, "Optimization of the Method of Auxiliary Sources (MAS), for scattering by an infinite cylinder under oblique incidence", Electromagnetics, vol. 25, no. 1, 2005, pp. 39-54

[6] N. L. Tsitsas, E. G. Alivizatos, H. T. Anastassiu and D. I. Kaklamani, "Optimization of the Method of Auxiliary Sources (MAS), for oblique incidence scattering by an infinite dielectric cylinder", Arch. für Elektrot. (Elect. Eng.), 89, 2007, pp. 353-361, DOI 10.1007/s00202-006-0019-1, http://dx.doi.org/10.1007/s00202006-0019-1.

[7] A. G. Kyurkchan, B. Y. Sternin and V. E. Shatalov, "Singularities of continuation of wave Fields", Phys. Uspechi, 39 (12), 1996, pp. 1221-1242.

[8] H. T. Anastassiu, A. T. Vouldis and G. K. Avdikos, "Optimization schemes for the Method of Auxiliary Sources applied to the scattering problem from circular-like metallic and dielectric objects", WSEAS Trans. Commun., vol. 4, no. 10, 2005, pp. 1138-1145.

[9] A. T. Vouldis, G. K. Avdikos and H. T. Anastassiu, "Preconditioning techniques for the method of auxiliary sources applied to geometries characterized as perturbations of a circle," Proc. EUCAP 2006, (ESA SP-626), Nice, France, November 2006.

[10] G. Fikioris, "On two types of convergence in the Method of Auxiliary Sources (MAS)", IEEE Trans. Antennas Propagat., vol. 54, no. 7, July 2006, pp. 2022-2033. 